Article

\title{
Identification of Candidate Genes and Pathways Associated with Obesity-Related Traits in Canines via Gene-Set Enrichment and Pathway-Based GWAS Analysis
}

\author{
Sunirmal Sheet ${ }^{\dagger}$, Srikanth Krishnamoorthy ${ }^{+}{ }^{+}$, Jihye Cha, Soyoung Choi and Bong-Hwan Choi * \\ Animal Genome \& Bioinformatics, National Institute of Animal Science, RDA, Wanju 55365, Korea; \\ sunirmal.micro@gmail.com (S.S.); kris87@korea.kr (S.K.); wischa91@korea.kr (J.C.); csy7pp@korea.kr (S.C.) \\ * Correspondence: bhchoi@korea.kr; Tel.: +82-10-8143-5164 \\ + These authors contributed equally.
}

Received: 10 October 2020; Accepted: 6 November 2020; Published: 9 November 2020

check for updates

Simple Summary: Obesity is a serious health issue and is increasing at an alarming rate in several dog breeds, but there is limited information on the genetic mechanism underlying it. Moreover, there have been very few reports on genetic markers associated with canine obesity. These studies were limited to the use of a single breed in the association study. In this study, we have performed a GWAS and supplemented it with gene-set enrichment and pathway-based analyses to identify causative loci and genes associated with canine obesity in 18 different dog breeds. From the GWAS, the significant markers associated with obesity-related traits including body weight (CACNA1B, C22orf39, U6, MYH14, PTPN2, SEH1L) and blood sugar (PRSS55, GRIK2), were identified. Furthermore, the gene-set enrichment and pathway-based analysis (GESA) highlighted five enriched pathways (Wnt signaling pathway, adherens junction, pathways in cancer, axon guidance, and insulin secretion) and seven GO terms (fat cell differentiation, calcium ion binding, cytoplasm, nucleus, phospholipid transport, central nervous system development, and cell surface) which were found to be shared among all the traits.

\begin{abstract}
The present study aimed to identify causative loci and genes enriched in pathways associated with canine obesity using a genome-wide association study (GWAS). The GWAS was first performed to identify candidate single-nucleotide polymorphisms (SNPs) associated with obesity and obesity-related traits including body weight and blood sugar in 18 different breeds of 153 dogs. A total of 10 and 2 SNPs were found to be significantly $\left(p<3.74 \times 10^{-7}\right)$ associated with body weight and blood sugar, respectively. None of the SNPs were identified to be significantly associated with obesity trait. We subsequently followed up the GWAS analysis with gene-set enrichment and pathway analyses. A gene-set with 1057, 1409, and 1243 SNPs annotated to 449, 933 and 820 genes for obesity, body weight, and blood sugar, respectively was created by sub-setting the GWAS result at a threshold of $p<0.01$ for the gene-set enrichment analysis. In total, 84 GO and 21 KEGG pathways for obesity, 114 GO and 44 KEGG pathways for blood sugar, 120 GO and 24 KEGG pathways for body weight were found to be enriched. Among the pathways and GO terms, we highlighted five enriched pathways (Wnt signaling pathway, adherens junction, pathways in cancer, axon guidance, and insulin secretion) and seven GO terms (fat cell differentiation, calcium ion binding, cytoplasm, nucleus, phospholipid transport, central nervous system development, and cell surface) that were found to be shared among all the traits. Our data provide insights into the genes and pathways associated with obesity and obesity-related traits.
\end{abstract}


Keywords: obesity; post-GWAS; gene-set enrichment; pathway analysis; functional annotation; genetic variants; blood sugar; body weight; canine

\section{Introduction}

Canine obesity is a global epidemic rising all over the world. Among dogs visiting veterinary practices, roughly $34-59 \%$ of dogs are reported to be overweight, with $5-20 \%$ obese [1-4]. The contemporary rise in obesity is triggered by lifestyle; however, the extensive inter-individual deviation in body mass index (BMI) witnessed even under shared environmental conditions can only be attributed to a genetic predisposition to the condition [5]. Genetic mutations within a single gene have been reported to have a large effect on obesity [6]. Inbreeding leading to reduced genetic diversity is also considered as a crucial factor associated with obesity. Inbreeding increases the prevalence of genetic defects because of the homozygosity of a large number of deleterious recessive alleles [7].

In addition, as in human beings, obesity causes several adverse consequences in dogs, such as cardiopulmonary disease, insulin resistance, diabetes, osteoarthritis, cancer, and other endocrine disorders $[4,8,9]$. Canines are considered to be obese when their body weight exceeds $30 \%$ of their optimal body weight [4]. Gaining extra body weight always precedes the onset of obesity [4]. In obese individual, adipocytes release elevated amounts of free fatty acids, cytokines (TNF- $\alpha$ ), leptin, glycerol, adiponectin, and several other factors that are involved in regulating insulin secretion, blood sugar level and body weight, which ultimately contributes to the development of insulin resistance [10-12]. Previously, many studies have reported that body weight, obesity, and blood sugar have a clear positive association [13-15]. The dog is also considered an important model for obesity research because of its clinical and molecular similarity with humans, but the knowledge about the genetic contribution to dog obesity remains insufficient.

Post-genome-wide association study (GWAS) analyses have been performed in several studies to discover obesity-related markers [16-18]. Previous association studies have revealed that polymorphism of POMC and TNF gene can affect the body weight and appetite in obesity [16,19]. A few other genes such as FTO, PPARG, BDNF, MC4R, and MC3R have been reported to be associated with obesity in canines [16-18]. Till now, only a few studies on the effect of polymorphism of canine genes on obesity have been published. On the other hand, polymorphism of more than 50 genes was reported to be associated with human obesity [4,20]. Therefore, the continuous search for important candidate canine genes associated with obesity is required. It will help to understand the etiology of obesity in canine. GWAS is a widely used method for studying the genetics of complex diseases [21]. GWAS have so far yielded a huge number of associations between single-nucleotide polymorphisms (SNPs) and complex diseases [21-23]. However, performing GWAS powerful enough is relatively difficult because of a few factors like small effect sizes of variants in complex disease, and required stringent statistics because of multiple testing problems. As a result, GWAS identifies only large effect sizes of variants [24]. In addition, GWAS does not capture multi-allelic QTL because of the bi-allelic nature of single nucleotide variants (SNVs) and does not account the fact that disease-associated genes or their products work together in a network [22-24]. Therefore, a robust GWAS analysis with the aim of detailed biological understanding is challenging. The solutions proposed to overcome these limitations are to focus on post-GWAS analyses such as functional gene and pathway enrichment analysis, protein-protein interaction network analysis, and translational medicine [22,24-26].

In the present study, we performed a case-control-based GWAS analysis using SNP genotyping data from Illumina CanineHD BeadChip array and supplemented it with gene-set and pathway-based functional analysis to detect significantly associated candidate genes and pathways with obesity, and related traits (blood sugar and body weight) in dogs. 


\section{Materials and Methods}

\subsection{Animals and Phenotype Assignment}

A total of 153 dogs from across South Korea were used in the present study and blood samples were collected from them by following relevant guidelines formulated by the Institutional Animal Care and Use Committee (IACUC) of the National Institute of Animal Science (NIAS, RDA, Wanju-gun, South Korea), and protocol consent was obtained for 'Development of early diagnosis technology for degenerative muscular skeleton system in special-purpose dog' project. All dogs were diagnosed individually by a veterinarian. Obesity traits were classified into a 5-point scoring system (1-5) using the body condition score (BCS) [27] as given in Table 1. BCS scores of 1 to 4 were used as controls and a score of 5 as a case for the case-control analysis of obesity. For case-control analysis of body weight trait, the dog of a particular breed having higher body weight, higher than the threshold suggested in America Kennel club standards, was selected as case and dogs falling within the suggested standards [28,29] were considered as control. The case-control analysis for blood sugar was distinguished based on the blood glucose concentration. Individuals with fasting blood glucose level over $120 \mathrm{mg} / \mathrm{dL}$ were considered as case. Dogs whose fasting blood glucose level is in the normal range (80-120 mg/dL) were considered as control. All dogs were kept under fasting for $12 \mathrm{~h}$ from their last meal before the blood sampling in the morning.

Table 1. Total dogs categorized based on body condition score for obesity case-control analysis.

\begin{tabular}{cccccc}
\hline Body Condition Score & $\mathbf{1}$ & $\mathbf{2}$ & $\mathbf{3}$ & $\mathbf{4}$ & $\mathbf{5}$ (Case) \\
\hline & Very thin & Underweight & Ideal body weight & Overweight & Obese \\
Number of animal & 7 & 11 & 98 & 11 & 29 \\
\hline
\end{tabular}

\subsection{Genomic DNA Extraction, SNP Genotyping and Quality Control}

Genomic DNA was extracted from blood samples of 153 dogs of 18 different breeds, using the DNeasy Blood and Tissue Kit (Qiagen, Valencia, CA, USA). The samples were genotyped on an Illumina CanineHD BeadChip (Illumina, San Diego, CA, USA) array, which contains 173,662 SNPs. Quality control (QC) was carried out with PLINK v.1.9 software [24] under the following criteria: minor allele frequency $<5 \%$, low genotyping call rate $<90 \%$, missing genotype calls $>10 \%$, Hardy-Weinberg equilibrium at $p<0.000001$. The final genotyping call rate was $98.5 \%$. Following QC filtering, 135,553 SNPs and 152 animals remained for further association analysis.

\subsection{Genome-Wide Association Analysis}

A genome-wide association study (GWAS) was performed using a logistic regression model implemented in PLINK v.1.9, to test the association between disease trait and allele in each SNP. Significant factors such as age (1-17), sex (85 females 68 males), and breed (18) were fitted in the GWAS statistical model for all the traits. We generated a total of 20 principal components (PCs); the eigenvalues of all the PCs were fit as co-variance to account for population stratification. The GWAS statistical model used was as follows:

$$
y=x \beta+z u+e
$$

where $y$ is a phenotype, $x$ and $\mathrm{z}$ are design matrices, $\beta$ is a vector of fixed effects, $\mathrm{u}$ is an additive genetic effect for each marker, and e is a vector of residual [30]. A Bonferroni-corrected threshold was applied to correct for multiple testing, and the genome-wide significance threshold was $p<3.74 \times 10^{-7}$ $(\sim 0.05 / 135,553)$. Manhattan and quantile-quantile (Q-Q) plots were generated using CMplot package in $R$ [24]. 


\subsection{Gene-Set Enrichment and Pathway Analysis}

We conducted gene-set enrichment and pathway analysis for each trait following the methods described by Dadousis et al. [24,25]. The markers were assigned to harboring genes or to genes within a flanking region of $5 \mathrm{~kb}$ up- and downstream of the SNP using SnpEff version 4.3 software [24]. A nominal $p<0.01$ was used to filter for SNPs from the GWAS analysis for gene-set enrichment and pathway analysis. The reason behind adopting a less stringent threshold for GWAS was to identify SNPs which do not reach the stringent significant thresholds set in GWAS analysis but still contributes to phenotypic variability. Furthermore, gene-set and pathway analyses obtained from GWAS provided additional knowledge about the complex relationships among genes and interrelated pathways which are likely to play role in obesity [31]. Merging less significant but connected SNPs we can understand well how these variants might be collectively related to our phenotypes of interest [31]. The SNP IDs, genes name assigned to SNPs was filtered from a variant call format (VCF) file that was previously mapped using SnpEff version 4.3 software. The Kyoto Encyclopedia of Genes and Genomes (KEGG) pathway database and Gene Ontology (GO) database under biological process and molecular function categories were used for functional annotation and enrichment analyses. Over-representation of genes in every GO terms and pathways were tested using a Fisher's exact test, which was conducted using the web-based gene-enrichment analysis tool, the Database for Annotation, Visualization, and integrated discovery (DAVID, http://david.abcc.ncifcrf.gov/) [32]. False discovery rate (FDR) correction $(<0.05)$ was applied to account for multiple testing [32]. The GO terms/KEGG category pathways with more than 10 and less than 1000 genes were analyzed for narrowing down the functional categories.

\section{Result and Discussion}

A polygenic complex disease such as obesity is generally controlled by additive genetic effects of a large number of genes which harbors several SNPs with small-effect size [24]. Some of these SNPs do not reach the stringent statistic thresholds, which are adapted to control for multiple testing problems in GWAS. Therefore, in this study, we included GO and pathways enrichment analyses, which were performed using the post-GWAS assigned genes (harboring essential SNPs) to understand the genetic contribution and biological significance of the functional SNPs on three traits (obesity, blood sugar, and body weight).

\subsection{Phenotypes}

In the current study, 85 females and 68 males between 1 and 17 years of age were used for analysis. As shown in Table 1, a total of 153 dogs are classified into 5 different BCS categories for case/control analysis of obesity trait. The dogs with a BCS score of 5 were designated as obese [27]. Therefore, we assigned this dog group as a case. In total, there were 29 cases and 124 controls for obesity trait. The total number of cases and controls for body weight was 28 and 125 . The overlap between the two categories was 28 individuals, which were both obese and cases for body weight. For blood sugar, it was 34 cases and 119 controls (Table 2). Earlier studies have reported that the medium-sized dogs are more likely to be obese than small-sized dogs or toy breed dogs $[27,33]$. In this study, most of the obese animals were also from the small category dogs (Pomeranian, Yorkshire Terrier, and Miniature Pinscher) or toy-sized (Maltese and Shihtzu) (Table 3). On the other hand, only a few cases were medium-sized dogs such as Beagles and Cocker Spaniel. Possibly, the small number of medium and large-sized dogs in our study is the reason for inconsistent result with the previously reported study $[27,33]$. 
Table 2. Number of cases and controls used in this study for each trait.

\begin{tabular}{cccc}
\hline Group & Number of Animals & Number of Control & Number of Case \\
\hline Obesity & 153 & $124($ BCS $1-4)$ & $29($ BCS 5$)$ \\
Body weight & 153 & 125 & $28^{*}$ \\
Blood sugar & 153 & $119(\leq 120 \mathrm{mg} / \mathrm{dL})$ & $34(\geq 120 \mathrm{mg} / \mathrm{dL})$ \\
\hline
\end{tabular}

* Dogs weighing over the America Kennel club standards were selected as cases; BCS—body condition score.

Table 3. Summary of phenotypes (including breed, number of male and female dogs investigated, and number of cases) used to perform GWAS in our analysis.

\begin{tabular}{cccccc}
\hline \multirow{2}{*}{ No. } & Breed Name & $\begin{array}{c}\text { Number of Dogs } \\
\text { Investigated }\end{array}$ & Obesity & Body Weight & Blood Sugar \\
\cline { 3 - 6 } & & 85 Females 68 Males & Case & Case & Case \\
\hline 1 & Beagle & 3 & 1 & 1 & 1 \\
2 & Bichon fris & 1 & 0 & 0 & 0 \\
3 & Chihuahua & 6 & 0 & 0 & 2 \\
4 & Cocker Spaniel & 8 & 1 & 1 & 1 \\
5 & Dachshund & 3 & 0 & 0 & 0 \\
6 & Doberman & 3 & 0 & 0 & 1 \\
7 & German Shepherd & 1 & 0 & 0 & 0 \\
8 & Golden Retriever & 1 & 0 & 0 & 7 \\
9 & Maltese & 40 & 8 & 7 & 1 \\
10 & Miniature Pinscher & 3 & 5 & 5 & 3 \\
11 & Mixed & 19 & 1 & 1 & 6 \\
12 & Parson Russell Terrier & 5 & 5 & 5 & 2 \\
13 & Pomeranian & 7 & 2 & 2 & 1 \\
14 & Poodle & 20 & 1 & 1 & 2 \\
15 & Schnauzer & 6 & 1 & 1 & 0 \\
16 & Shih tzu & 10 & 2 & 2 & 4 \\
17 & Spitz & 5 & 0 & 0 & 2 \\
18 & Yorkshire Terrier & 12 & 2 & & \\
\hline
\end{tabular}

\subsection{Genome-Wide Association Study}

To find significant loci associated with the three traits (obesity, body weight, and blood sugar), we performed GWAS with 133,553 SNPs which passed our filtering criteria. The Q-Q plot of the association study indicated a significant clear deviation for blood sugar $(\lambda$ value $=0.961)$ and body weight $(\lambda$ value $=0.963)$, but, for obesity, there was no obvious deviation $(\lambda$ value $=1.091)$ (Figures $1-3)$. The $p$-value obtained from the GWAS was plotted as Manhattan plots (Figures 1-3). Our result showed that 2 and 7 SNPs were significantly associated to blood sugar and body weight, respectively, at the genome-wide significance level of $p<3.74 \times 10^{-7}$ (i.e., Bonferroni corrected threshold $(0.05 / 133,553$ ). The candidate SNPs associated with blood sugar were on chromosomes 25 and 12; whereas for body weight they were on chromosome 9, 26, 37, 1, 39, and 7 (Table 4). Moreover, none of the SNPs were found to be significant $\left(p<3.74 \times 10^{-7}\right)$ for obesity. 


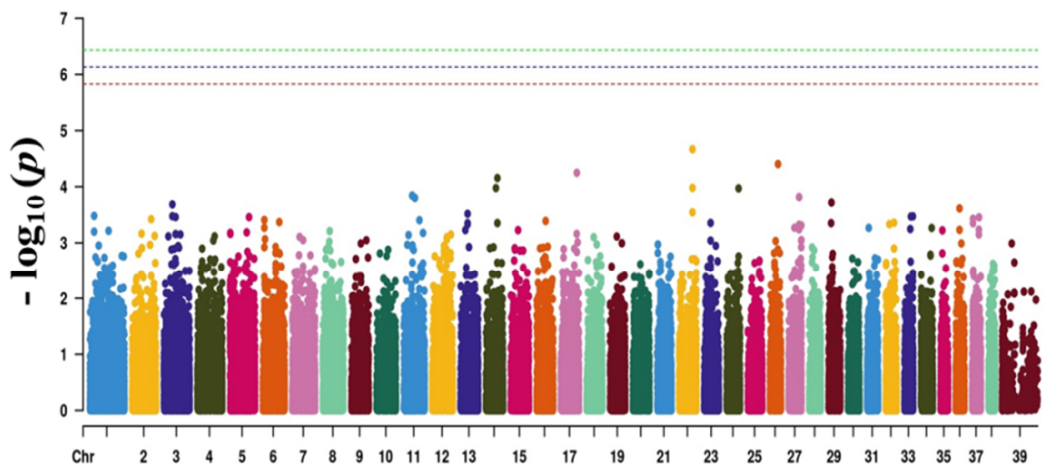

Chromosome

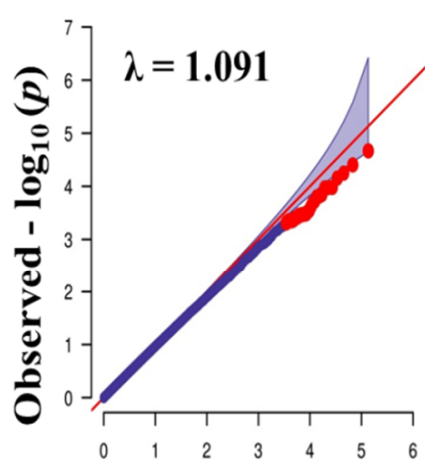

Expected $-\log _{10}(p)$

Figure 1. Manhattan plots showing the distribution of $p$-values of single nucleotide polymorphism (SNP) markers associating with obesity. Greenline designates the genome-wide significant threshold level of $p<3.74 \times 10^{-7}$, blue line designates suggestive threshold level of $p<7.48 \times 10^{-7}$, and red line designates suggestive threshold level of $p<5.82 \times 10^{-6}$. The quantile-quantile (Q-Q) plot of the GWAS is shown on the right side. GWAS-genome-wide association study.

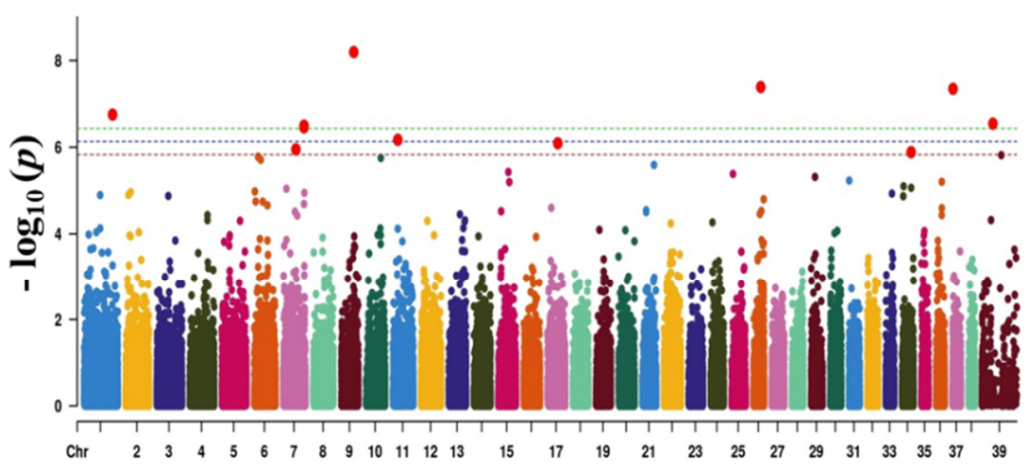

Chromosome

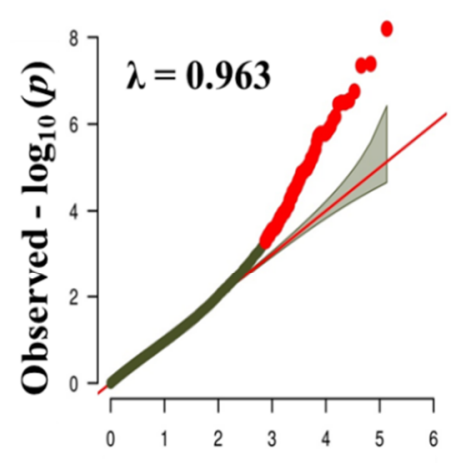

Expected $-\log _{10}(p)$

Figure 2. Manhattan plot showing the distribution of $p$-values of single nucleotide polymorphism (SNP) markers associated with body weight. Greenline designates the genome-wide significant threshold level of $p<3.74 \times 10^{-7}$, blue line designates suggestive threshold level of $p<7.48 \times 10^{-7}$, and red line designates suggestive threshold level of $p<5.82 \times 10^{-6}$. The quantile-quantile (Q-Q) plot of the GWAS is shown on the right side. GWAS - genome-wide association study.

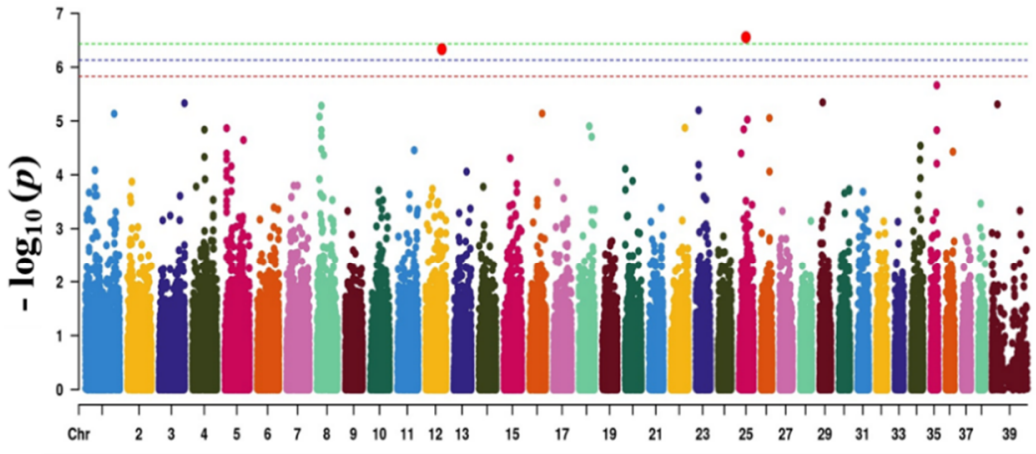

Chromosome

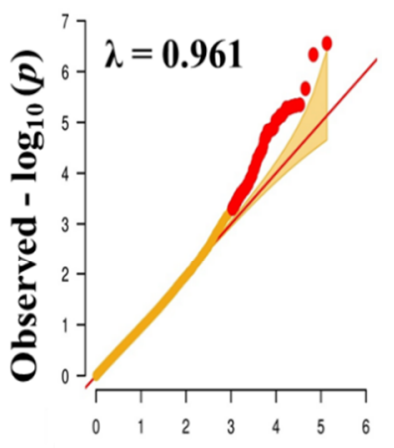

Expected $-\log _{10}(p)$

Figure 3. Manhattan plot showing the distribution of $p$-values of single nucleotide polymorphism (SNP) markers associating with blood sugar. Greenline designates the genome-wide significant threshold level of $p<3.74 \times 10^{-7}$, blue line designates suggestive threshold level of $p<7.48 \times 10^{-7}$, and red line designates suggestive threshold level of $p<5.82 \times 10^{-6}$. The quantile-quantile (Q-Q) plot of the GWAS is shown on the right side. GWAS - genome-wide association study. 
Table 4. Top SNPs associated with obesity, body weight, and blood sugar in dogs.

\begin{tabular}{ccccccc}
\hline Trait & SNP ID & Chr & Position & Freq & Gene & Type \\
\hline Body weight & BICF2P1168261 & 9 & 47831552 & $6.38 \times 10^{-9}$ & CACNA1B & Intron variant \\
& G1314f25S201 & 26 & 29766195 & $4.09 \times 10^{-8}$ & C22orf39 & Synonymous variant \\
& BICF2P940718 & 37 & 5443556 & $4.50 \times 10^{-8}$ & Intergenic region \\
& BICF2P407675 & 1 & $1.06 \times 10^{8}$ & $1.77 \times 10^{-7}$ & MYH14 & Intron variant \\
& BICF2P247463 & 39 & 39876923 & $2.86 \times 10^{-7}$ & - & - \\
& BICF2P1124008 & 7 & 78278707 & $3.20 \times 10^{-7}$ & PTPN2 & Intergenic region \\
& BICF2S23242598 & 7 & 78365053 & $3.20 \times 10^{-7}$ & SEH1L & Intron variant \\
Blood sugar & BICF2P1418953 & 25 & 27416887 & $2.78 \times 10^{-7}$ & PRSS55 & Downstream_gene_ \\
& BICF2G630121162 & 12 & 60529545 & $4.64 \times 10^{-7}$ & GRIK2 & Intergeniant region \\
\hline
\end{tabular}

The two SNPs significantly associated with blood sugar, BICF2P1418953 (downstream gene variant) and BICF2G630121162 (intragenic region) were annotated to PRSS55 and GRIK2 genes, respectively (Table 4). The serine protease 55 (PRSS55) belongs to the member of a group of membrane-anchored chymotrypsin (S1)-like serine protease that has been proven to play a key role in maintaining homeostasis [34-36]. Obesity is directly attributable to homeostasis imbalance [37]. Furthermore, glutamate receptor ionotropic receptor kainite type subunit 2 (GRIK2) is involved in the majority of glutametergic neurotransmission, which has been suggested to take part in the onset of obesity by regulating appetite $[38,39]$.

In addition, BICF2P1168261 (intron variant), G1314f25S201 (synonymous variant), BICF2P940718 (intergenic region), BICF2P407675 (intron variant), BICF2P1124008 (intergenic region), and BICF2S23242598 (intron variant), which were significantly associated with body weight, were located in protein-coding genes Calcium Voltage-Gated Channel Subunit Alpha1 B (CACNA1B), U6 (U6 non-coding small nuclear RNA), Myosin Heavy Chain 14 (MYH14), Protein Tyrosine Phosphatase Non-receptor Type 2 (PTPN2), and SEH1 like Nucleoporin $(S E H 1 L)$, respectively. The most significantly associated amongst these was BICF2P116826, harbored by calcium voltage-gated channel subunit alpha1 B (CACNA1B), a member of the voltage-gated calcium channels [40]. The voltage-gated calcium channels are the main regulators of calcium signaling in adipogenesis, which eventually contribute to obesity [40-42]. In addition, $C A C N A 1 B$ gene has been previously reported to be a therapeutic target for diseases such as lung cancer, prostate cancer and breast cancer [40]. Moreover, the gene, $C A C N A 1 B$ was noticed to be significantly enriched in several other pathways associated with the traits evaluated in this study, suggesting a significant connection with obesity disease. Therefore, we suggest that the CACNA1B gene can be a good candidate marker. The body weight trait in dogs has been examined in several studies based on the GWAS approach [29]. Though no previously reported genes/markers were found to be associated in our study, some of the candidate markers/genes identified in this study were on chromosome $X$ (indicated as chr 39 in this study), which was previously reported to harbor genes/markers found to have a significant effect on body weight in dogs [29]. The lack of overlapping genes/marker with previous reports may be due to use of different breeds in this study, and the inclusion of higher number of large breed dogs, and differences in phenotype assignment.

In addition, compared to obesity in humans, there are only limited reports on genetic markers associated with canine obesity. In humans, more than 220 obesity-related genetic markers have been documented throughout the last decade [16]. Among these, FTO, BDNF, MC4R, PCSK1, MC3R, and PPARG genes were considered as promising candidate genes for canine obesity [16-18,43]. Recently, Mankowska et al. reported another candidate gene, $P O M C$, which showed a strong association with weight and appetite in obesity-prone Labrador retrievers [19,20]. In the current study, several different candidate genes were found; however, none were common to those previously reported. However, the identified markers could serve as a good candidate for further studies. 


\subsection{Gene-Set Enrichment and Pathway Analysis}

GWAS was complemented with a functional enrichment analysis to explore the significant pathways/GO terms associated with obesity and other traits. It showed the enrichment of annotated gene-sets which worked together in a network to carry out specific molecular processes. Out of 135,554 SNPs used in GWAS, 91,779 were located within mapped genes or the $5 \mathrm{~Kb}$ flanking regions of the mapped genes. A total of 1057, 1409, and 1243 SNPs showed an association for obesity, body weight, and blood sugar at a nominal threshold of $p<0.01$, respectively (Supplementary Table S1). These SNPs were mapped to 449,933 , and 820 unique genes for obesity, body weight, and blood sugar trait, respectively (Supplementary Table S1). Subsequently, gene-set and pathway analysis were performed using DAVID. A total of 84, 114, and 120 GO terms and 21, 44, and 24 KEGG pathways were identified to be enriched for obesity, blood sugar, and body weight, respectively (Supplementary Table S2). Out of the total enriched GO terms and KEGG pathways, the top 5 significantly enriched GO terms and pathways are presented in Table 5. The pathways commonly enriched in all the three traits (obesity, blood sugar, and body weight) (Table 6) were: cfa04310 —Wnt signaling pathway, cfa04520—adherens junction, cfa05200 — pathways in cancer, cfa04360 - axon guidance, and cfa04911—insulin secretion. Likewise, a total of 7 GO terms (GO:0045444-fat cell differentiation, GO:0005509—calcium ion binding, GO:0005737—cytoplasm, GO:0005634—nucleus, GO:0015914—phospholipid transport, GO:0007417-central nervous system development, GO:0009986 — cell surface) were found to be commonly enriched in all the three evaluated traits (Table 6).

\subsubsection{Wnt Signaling, Adherens Junction, and Axon Guidance Pathways}

Wnt signaling is a crucial pathway for adipogenesis. This pathway is involved in the development of obesity-induced insulin resistance [44,45]. Wnt signaling has been proposed as a targeting pathway to fight obesity due to its critical role in the development of white adipose tissue and brown adipose tissue [46]. Moreover, an important transcription factor of Wnt signaling was previously identified as a candidate gene for type 2 diabetes [47]. In addition, adherens junction and axon guidance were significantly enriched in obesity, blood sugar, and body weight. An adherens junction is an intercellular junction and is important for epithelial adhesion. Adherens junction plays a key role in the regulation of insulin secretion using cadherin protein which ultimately reveals strong association with blood glucose level, obesity, and diabetes [48]. Amongst the enriched genes in these pathways, Wnt family member 1 (WNT1) was previously reported to be associated with obesity and the importance of this gene in obesity was also highlighted $[46,49]$.

\subsubsection{Cancer-Related Pathways}

The pathways in cancer (cfa05200) were significantly enriched in all the three traits including obesity, blood sugar, and body weight. Apart from that other significantly enriched cancer-related pathways, including, endometrial cancer (cfa05213), melanoma (cfa05218), prostate cancer (cfa05215), proteoglycans in cancer (cfa05205) were detected with some pathways being in common among all traits. The association between cancer and obesity is well documented, obesity increases the risk of various cancers in the human population [50,51]. Further, Lim et al. reported that the increased expression of obesity-associated molecules increases the risk of development of mammary gland tumors in obese female canines [51]. These pathways are enriched with numerous genes and in fact, the mutations in a few key genes have been described to affect obesity or blood sugar trait [12,49,52-54]. In total, 64 genes were significantly enriched in cancer-related pathways and several significant genes such as cyclic AMP-responsive element-binding protein 5 (CREB5) [55], AKT serine/threonine kinase 3 (AKT3) [53], cyclin D1 (CCND1) [12,52], Wnt family member 1 (WNT1) [49,54] were identified to be associated with blood sugar and obesity traits. 
Table 5. Top 5 Gene Ontology and KEGG pathways significantly enriched using genes associated with obesity, body weight, and blood sugar.

\begin{tabular}{|c|c|c|c|c|c|c|c|}
\hline Trait & Category & Term_ID & Term & Count & $\%$ & $p$-Value & Genes \\
\hline \multirow[t]{10}{*}{ Obesity } & KEGG_PATHWAY & cfa04360 & Axon guidance & 10 & 0.015278 & $7.15 \times 10^{-4}$ & $\begin{array}{c}\text { DCC, MAPK1, NGEF, EPHA7, CXCR4, GNAI1, ROBO1, } \\
\text { UNC5D, LRRC4C, EPHB1 }\end{array}$ \\
\hline & KEGG_PATHWAY & cfa04550 & $\begin{array}{l}\text { Signaling pathways } \\
\text { regulating pluripotency of } \\
\text { stem cells }\end{array}$ & 10 & 0.015278 & 0.0015 & $\begin{array}{c}\text { MAPK1, FGFR1, BMP2, ONECUT1, FZD1, FZD3, } \\
\text { WNT11, FZD2, ZFHX3, KLF4 }\end{array}$ \\
\hline & KEGG_PATHWAY & cfa05200 & Pathways in cancer & 18 & 0.0275 & 0.0016 & $\begin{array}{l}\text { DCC, FGFR1, BMP2, COL4A1, BRAF, GNAI1, FGF9, } \\
\text { RUNX1T1, FZD1, FZD3, FZD2, GLI3, CTNNA3, } \\
\text { CTNNA2, LAMA2, MAPK1, CXCR4, WNT11 }\end{array}$ \\
\hline & KEGG_PATHWAY & cfa04520 & Adherens junction & 7 & 0.010695 & 0.0026 & $\begin{array}{c}\text { MAPK1, FGFR1, TJP1, PTPRM, SSX2IP, } \\
\text { CTNNA3, CTNNA2 }\end{array}$ \\
\hline & KEGG_PATHWAY & cfa05217 & Basal cell carcinoma & 6 & 0.009167 & 0.0040 & BMP2, FZD1, FZD3, WNT11, FZD2, GLI3 \\
\hline & GOTERM_MF_DIRECT & GO:0016874 & Ligase activity & 7 & 1.682692 & $3.74 \times 10^{-4}$ & $\begin{array}{l}\text { HECW2, UBE3A, SUCLG2, HECTD3, SIAH1, } \\
\text { SMURF1, NEDD4L }\end{array}$ \\
\hline & GOTERM_BP_DIRECT & GO:0045892 & $\begin{array}{l}\text { Negative regulation of } \\
\text { transcription, } \\
\text { DNA-templated }\end{array}$ & 13 & 3.125 & 0.0031 & $\begin{array}{c}\text { RBFOX2, BCLAF1, RUNX1T1, FZD1, PAX2, CBFA2T3, } \\
\text { ADIPOQ, GAS6, ZSCAN10, LHX1, ATP8B1, } \\
\text { POU3F3, WNT11 }\end{array}$ \\
\hline & GOTERM_BP_DIRECT & GO:0060022 & Hard palate development & 3 & 0.721154 & 0.0034 & FZD1, FZD2, MMP25 \\
\hline & GOTERM_BP_DIRECT & GO:0034115 & $\begin{array}{l}\text { Negative regulation of } \\
\text { heterotypic cell-cell adhesion }\end{array}$ & 3 & 0.721154 & 0.0056 & $A P O A 1, A D I P O Q, K L F 4$ \\
\hline & GOTERM_BP_DIRECT & GO:0002062 & Chondrocyte differentiation & 5 & 1.201923 & 0.0057 & SNX19, FGFR1, BMP2, FGF9, NFIB \\
\hline \multirow[t]{7}{*}{$\begin{array}{l}\text { Body } \\
\text { weight }\end{array}$} & KEGG_PATHWAY & cfa05033 & Nicotine addiction & 8 & 0.900900 & 0.0021 & $\begin{array}{c}\text { GABRG3, GRIA2, GRIA1, GABRB1, GABRA5, GRIN2A, } \\
\text { GRIN3A, CACNA1B }\end{array}$ \\
\hline & KEGG_PATHWAY & cfa04080 & $\begin{array}{l}\text { Neuroactive } \\
\text { ligand-receptor interaction }\end{array}$ & 23 & 2.590090 & 0.0049 & $\begin{array}{c}\text { GABRG3, GLRA1, GRIK2, GABRB1, OPRK1, GRIN3A, } \\
\text { P2RY6, GRIA2, GRIA1, NMUR2, HRH4, ADRA1A, } \\
\text { NMBR, PRL, CHRNE, PTAFR, GRID1, GHR }\end{array}$ \\
\hline & KEGG_PATHWAY & cfa05206 & MicroRNAs in cancer & 13 & 1.463963 & 0.0268 & $\begin{array}{l}\text { KIF23, PDGFA, SOCS1, MET, BMPR2, PIM1, TP63, } \\
\text { ZEB1, IRS1, PDCD4, CCND1, CDKN2A, DNMT1 }\end{array}$ \\
\hline & KEGG_PATHWAY & cfa04022 & $\begin{array}{l}\text { cGMP-PKG } \\
\text { signaling pathway }\end{array}$ & 14 & 1.576576 & 0.0293 & $\begin{array}{c}\text { EDNRA, KCNU1, KCNMB4, PLCB4, TRPC6, ATP2A3, } \\
\text { GTF2IRD1, ADRA1A, CREB5, NOS3, PLCB1, CACNA1D, } \\
\text { IRS1, KCNMB2 }\end{array}$ \\
\hline & KEGG_PATHWAY & cfa04310 & Wnt signaling pathway & 12 & 1.351351 & 0.0394 & $\begin{array}{c}\text { DKK2, MAP3K7, CCND1, PLCB4, DKK1, VANGL1, } \\
\text { PRICKLE1, MMP7, SIAH1 }\end{array}$ \\
\hline & GOTERM_MF_DIRECT & GO:0004725 & $\begin{array}{l}\text { Protein tyrosine } \\
\text { phosphatase activity }\end{array}$ & 15 & 1.689189 & $5.27 \times 10^{-5}$ & $\begin{array}{c}\text { PTPRB, CDC14A, PTPN2, CDC14B, EPM2A, DUSP10, } \\
\text { PTPN13, PTPRT, PTPRU, EYA3, EYA4, EYA1, DUSP26, } \\
\text { UBASH3B, PTPN1 }\end{array}$ \\
\hline & GOTERM_BP_DIRECT & GO:0045444 & Fat cell differentiation & 12 & 1.3513513 & $1.90 \times 10^{-4}$ & $\begin{array}{l}\text { BBS2, METTL8, CCND1, FAM120B, SMAD6, BBS9, } \\
\text { FFAR2, SOCS1, OSBPL11, TTC8, PIAS1, PLCB1 }\end{array}$ \\
\hline
\end{tabular}


Table 5. Cont

\begin{tabular}{|c|c|c|c|c|c|c|c|}
\hline Trait & Category & Term_ID & Term & Count & $\%$ & $p$-Value & \multirow{14}{*}{$\begin{array}{c}\text { Genes } \\
\text { GLIS3, ACHE, SYT4, RAB3GAP1, NOS3, CDK5RAP2, } \\
\text { JAKMIP2, OLFM3, GOLM1, TERF2, CDK13, KLF5, } \\
\text { NMNAT2, MSH6, CLN3, PLD1, MYO6, DNM1L, } \\
\text { CCDC88A, LYN, ACO1, BEND5, GOLIM4, PKDCC, } \\
\text { NMT2, ATF6, DUSP26, CPE, BACE2, SULF1, DYM, } \\
\text { RAB14, SGCE, CWC22, EXT1 } \\
\text { CADM1, CLSTN2, SDK2, PCDH15, PCDH17, CDH8, } \\
\text { CDH13, DSG2, CDH18, FAT1, CDH19, FAT2, } \\
\text { CDH26, KIRREL3 } \\
\text { GLRA1, GRIK2, DENND1A, GDPD5, GRIN3A, KLHL1, } \\
\text { ALCAM, SEZ6L2, APOB, BRINP1, CPE, GRIA1, PSEN2, } \\
\text { RAPGEF2, BRINP3, CACNA1B } \\
\text { ACTB, THRB, ATP1A1, RCAN2, PLCB3, CCND1, PLCB4, } \\
\text { DIO2, GSK3B, PLCG2, PRKACB, PLCB1, AKT3, PIK3R1 } \\
\text { IRS2, E2F3, MCL1, MMP16, CDK6, ZEB2, ZEB1, PRKCE, } \\
\text { TIMP3, RPS6KA5, CCNE1, CCND1, PLCG2, } \\
\text { DNMT1, ZFPM2 } \\
\text { FHIT, CCND1, E2F3, PLCG2, CDK6, EGF, AKT3, } \\
\text { PIK3R1, EML4 } \\
\text { WNT1, PLCB3, PLCB4, GSK3B, MITF, EDN1, FZD1, } \\
\text { KITLG, PRKACB, PLCB1, WNT7A, CALM1 } \\
\text { PLCB3, IL1R1, PLCB4, PLCG2, IL1RAP, PLA2G6, } \\
\text { PRKCH, PRKACB, PRKCE, PLCB1, PIK3R1, CALM1 } \\
\text { ELP3, IRS2, BMP2, LYN, EDN1, HGF, DAB2, SEMA6D, } \\
\text { FOXF1, SEMA3C, COL1A1, PDGFD, PAK1, } \\
\text { PIK3R1, CSF1R } \\
\text { FRK, THRB, MITF, EDN1, ZEB2, PRDM16, EPC1, REL, } \\
\text { FOXF1, PRMT6, ETV6, DLG1, SIM2, ALX1, TBL1XR1, } \\
\text { BMP2, ASXL1, ZHX2, LMCD1, SMYD2, SHOX2, } \\
\text { HDAC4, CCND1, DKK1, DUSP26, PDE2A, HOPX, } \\
\text { TFAP2B, DNMT1, RIPPLY2, BMP6 } \\
\text { CYB5R4, CAV2, GALNT1, RAB3C, PKHD1, SLC39A12, } \\
\text { PINK1, ARFGEF1, SLC11A2, APP, BDNF, ECE1, PTK2B, } \\
\text { FAT1, TMEM192, CDK5RAP2, DLG1, PTPRM, LYN, } \\
\text { STC2, PRKCE, PDE2A, VAMP8, GSK3B, CYFIP2, } \\
\text { AKAP6, SPAST } \\
\text { EPHA5, SEMA6A, ZNF280D, KIF5B, ANK3, ROBO1, } \\
\text { SEMA3C, ETV1, RELN, UNC5D, CSF1R } \\
\text { SOX10, EDN3, SEMA6A, SEMA6D, SEMA3C, KITLG, } \\
\text { ZEB2, ALX1 }\end{array}$} \\
\hline \multirow{13}{*}{$\begin{array}{l}\text { Blood } \\
\text { sugar }\end{array}$} & GOTERM_CC_DIRECT & GO:0005794 & Golgi apparatus & 43 & 4.8423423 & $3.54 \times 10^{-4}$ & \\
\hline & GOTERM_BP_DIRECT & GO:0007156 & $\begin{array}{c}\text { Homophilic cell adhesion via } \\
\text { plasma membrane } \\
\text { adhesion molecules }\end{array}$ & 14 & 1.5765765 & $4.51 \times 10^{-4}$ & \\
\hline & GOTERM_CC_DIRECT & GO:0043025 & Neuronal cell body & 16 & 1.8018018 & $7.20 \times 10^{-4}$ & \\
\hline & KEGG_PATHWAY & cfa04919 & $\begin{array}{l}\text { Thyroid hormone } \\
\text { signaling pathway }\end{array}$ & 14 & 1.837270341 & $8.07 \times 10^{-4}$ & \\
\hline & KEGG_PATHWAY & cfa05206 & MicroRNAs in cancer & 15 & 1.968503937 & 0.0019 & \\
\hline & KEGG_PATHWAY & cfa05223 & Non-small cell lung cancer & 9 & 1.181102362 & 0.0023 & \\
\hline & KEGG_PATHWAY & cfa04916 & Melanogenesis & 12 & 1.57480315 & 0.0026 & \\
\hline & KEGG_PATHWAY & cfa04750 & $\begin{array}{l}\text { Inflammatory mediator } \\
\text { regulation of TRP channels }\end{array}$ & 12 & 1.57480315 & 0.0031 & \\
\hline & GOTERM_MF_DIRECT & GO:0004222 & $\begin{array}{l}\text { Metalloendopeptidase } \\
\text { activity }\end{array}$ & 15 & 1.968504 & 0.0011 & \\
\hline & GOTERM_BP_DIRECT & GO:0030335 & $\begin{array}{l}\text { positive regulation of } \\
\text { cell migration }\end{array}$ & 31 & 4.068241 & 0.0017 & \\
\hline & GOTERM_BP_DIRECT & GO:0000122 & $\begin{array}{l}\text { negative regulation of } \\
\text { transcription from RNA } \\
\text { polymerase II promoter }\end{array}$ & 27 & 3.543307 & 0.0017 & \\
\hline & GOTERM_CC_DIRECT & GO:0048471 & $\begin{array}{l}\text { perinuclear region } \\
\text { of cytoplasm }\end{array}$ & 11 & 1.44357 & 0.0039 & \\
\hline & GOTERM_BP_DIRECT & GO:0007411 & axon guidance & 8 & 1.049869 & 0.0041 & \\
\hline
\end{tabular}


Table 6. The significantly enriched GO terms and KEGG pathways shared amongst obesity, body weight, and blood sugar.

\begin{tabular}{|c|c|c|c|}
\hline Category & Term_ID & Term & Genes $^{a}$ \\
\hline GOTERM_BP_DIRECT & GO:0045444 & Fat cell differentiation & $A Q P 1$ \\
\hline GOTERM_MF_DIRECT & GO:0005509 & Calcium ion binding & CACNA1B, GRIK2 \\
\hline GOTERM_CC_DIRECT & GO:0005737 & Cytoplasm & ROBO1, LDHB \\
\hline GOTERM_CC_DIRECT & GO:0005634 & Nucleus & ATP2B1, CSRNP3, CSRNP2 \\
\hline GOTERM_BP_DIRECT & GO:0015914 & Phospholipid transport & PCTP \\
\hline GOTERM_BP_DIRECT & GO:0007417 & $\begin{array}{c}\text { Central nervous system } \\
\text { development }\end{array}$ & CHD7 \\
\hline GOTERM_CC_DIRECT & GO:0009986 & Cell surface & IL1R1, ROBO1, WNT1 \\
\hline KEGG_PATHWAY & $\mathrm{cfa} 04310$ & Wnt signaling pathway & WNT11, MAP3K7, WNT7A \\
\hline KEGG_PATHWAY & $\mathrm{cfa} 04520$ & Adherens junction & MAPK1, CTNNA2, MAP3K7 \\
\hline KEGG_PATHWAY & cfa05200 & Pathways in cancer & $\begin{array}{c}\text { CCND1, FZD1, FZD3 } \\
\text { FZD2, CTNNA1 }\end{array}$ \\
\hline KEGG_PATHWAY & cfa04360 & Axon guidance & MAPK1, ROBO1 \\
\hline KEGG_PATHWAY & cfa04911 & Insulin secretion & CACNA1B, CACNA1D, PLCB4 \\
\hline
\end{tabular}

${ }^{a}$ Enriched genes in KEGG pathways and GO shared among three traits, GO-Gene Ontology, KEGG-Kyoto Encyclopedia of Genes and Genomes.

\subsubsection{Insulin Secretion Pathway}

Another important pathway enriched with genes harboring SNPs associated with obesity was insulin secretion (cfa04911). This pathway was enriched with 7 genes in total. Insulin secretion occurs in pancreatic $\beta$ cells and it triggers the fusion of insulin-containing granules with the cell membrane [10]. Insulin secretion is an integral part of blood glucose levels control system. [10]. The positive association between obesity and insulin has been investigated for decades $[10,11]$. Obesity-associated with insulin resistance is a major risk factor of type 2 diabetes. However, insulin also plays a vital role in obesity by inducing ATP production in mitochondria to prevent $5^{\prime}$ AMP-activated protein kinase (AMPK) activity during hyperinsulinemia condition [56]. Recently, an important link between the insulin level and obesity was reported: diet-induced hyperinsulinemia assisted by glucagon hormone is an obligatory factor for obesity [11].

\subsubsection{Other Enriched Gene Ontology Terms}

We have highlighted seven important GO terms, which were significantly enriched in all three traits. These included fat cell differentiation, calcium ion binding, cytoplasm, nucleus, phospholipid transport, central nervous system development, and cell surface. The fat cell differentiation or adipocyte formation plays a crucial role in adipogenesis during obesity [46]. Calcium is an important ion for normal physiological functioning and calcium ion binding is involved in calcium signaling pathways which have been shown as key pathways in the regulation of obesity in many studies [40-42,57]. The multiple calcium signaling pathways play a most important role in the biological clock, neuronal excitability, and intestinal microbial activity for regulating the food intake and adipocyte metabolism, which subsequently reduces the occurrence of obesity [57]. The genes enriched included CACNA1B, which also harbored the significantly associated SNP BICF2P116826, encoding calcium voltage-gated channel subunit alpha1 B, which is associated with adipogenesis [40-42] and most importantly, it was identified as a candidate gene for body weight trait in our association study. Furthermore, the relation of phospholipid transport and obesity has been suggested to play a vital role in lipoprotein metabolism by transferring the phospholipids from triglyceride-rich lipoproteins to high-density lipoproteins [58].

\section{Conclusions}

The present study is the first to report about a post-GWAS analyses approach to prioritize the identification of genetic loci, pathways, and genes underlying molecular mechanisms of canine obesity and related traits such as body weight and blood sugar in multi-breed dogs. We have identified 
several significant candidate genes associated with obesity-related traits; in particular, CACNA1B gene harboring SNP BICF2P116826 could be a possible candidate gene for canine obesity. The gene-set and pathway analyses revealed five shared pathways, (Wnt signaling pathway, adherens junction, pathways in cancer, axon guidance, and insulin secretion) and seven GO terms to be associated with all evaluated traits, which probably explain that obesity is a polygenic trait. Overall, our results provide clues for identification of candidate SNPs and genes which have a significant impact on the etiology of canine obesity.

Supplementary Materials: The following are available online at http://www.mdpi.com/2076-2615/10/11/2071/s1, Table S1: List of all SNPs $(p<0.01)$ associated with obesity, body weight, and blood sugar that were used for gene-set and pathway analysis, Table S2: List of all Gene Ontology and KEGG pathways significantly enriched using genes associated with obesity, body weight, and blood sugar.

Author Contributions: Conceptualization, S.S., S.K., and B.-H.C.; methodology, S.S., S.K., J.C., and B.-H.C.; software, J.C., S.C; validation, S.S., S.K., and B.-H.C.; formal analysis, S.S. and S.C.; investigation, B.-H.C.; resources, B.-H.C. and J.C.; data curation, S.S., S.K.; writing-original draft preparation, S.S.; writing-review and editing, S.S. and S.K.; visualization, S.S., S.K.; supervision, S.K., B.-H.C.; project administration, B.-H.C.; funding acquisition, B.-H.C. All authors have read and agreed to the published version of the manuscript.

Funding: This research was supported by the funds from AGENDA project (grant no. PJ012833022019) of the National Institute of Animal Science, Rural Development Administration, Republic of Korea.

Conflicts of Interest: The authors declare that they have no conflict of interest. The funders had no role in the design of the study; in the collection, analyses, or interpretation of data; in the writing of the manuscript, or in the decision to publish the results.

\section{References}

1. McGreevy, P.D.; Thomson, P.C.; Pride, C.; Fawcett, A.; Grassi, T.; Jones, B. Prevalence of obesity in dogs examined by Australian veterinary practices and the risk factors involved. Vet. Rec. 2005, 156, 695-702. [CrossRef] [PubMed]

2. Colliard, L.; Ancel, J.; Benet, J.-J.; Paragon, B.-M.; Blanchard, G. Risk Factors for Obesity in Dogs in France. J. Nutr. 2006, 136, 1951S-1954S. [CrossRef] [PubMed]

3. Courcier, E.A.; Thomson, R.M.; Mellor, D.J.; Yam, P.S. An epidemiological study of environmental factors associated with canine obesity. J. Small Anim. Pr. 2010, 51, 362-367. [CrossRef] [PubMed]

4. Switonski, M.; Mankowska, M. Dog obesity-The need for identifying predisposing genetic markers. Res. Vet. Sci. 2013, 95, 831-836. [CrossRef] [PubMed]

5. Saeed, S.; Arslan, M.; Froguel, P. Genetics of Obesity in Consanguineous Populations: Toward Precision Medicine and the Discovery of Novel Obesity Genes. Obesity 2018, 26, 474-484. [CrossRef] [PubMed]

6. Wang, K.; Li, W.-D.; Zhang, C.K.; Wang, Z.; Glessner, J.T.; Grant, S.F.A.; Zhao, H.; Hakonarson, H.; Price, R.A. A Genome-Wide Association Study on Obesity and Obesity-Related Traits. PLoS ONE 2011, 6, e18939. [CrossRef]

7. Rudan, I.; Rudan, D.; Campbell, H.; Carothers, A.; Wright, A.; Smolej-Narancic, N.; Janicijevic, B.; Jin, L.; Chakraborty, R.; Deka, R.; et al. Inbreeding and risk of late onset complex disease. J. Med Genet. 2003, 40, 925-932. [CrossRef]

8. Mao, J.; Xia, Z.; Chen, J.; Yu, J. Prevalence and risk factors for canine obesity surveyed in veterinary practices in Beijing, China. Prev. Vet. Med. 2013, 112, 438-442. [CrossRef]

9. Yam, P.; Butowski, C.; Chitty, J.; Naughton, G.; Wiseman-Orr, M.; Parkin, T.; Reid, J. Impact of canine overweight and obesity on health-related quality of life. Prev. Vet. Med. 2016, 127, 64-69. [CrossRef]

10. Fu, Z.; Gilbert, E.R.; Liu, D. Regulation of Insulin Synthesis and Secretion and Pancreatic Beta-Cell Dysfunction in Diabetes. Curr. Diabetes Rev. 2013, 9, 25-53. [CrossRef]

11. Lee, Y.; Berglund, E.D.; Yu, X.; Wang, M.-Y.; Evans, M.R.; Scherer, P.E.; Holland, W.L.; Charron, M.J.; Roth, M.G.; Unger, R.H. Hyperglycemia in rodent models of type 2 diabetes requires insulin-resistant alpha cells. Proc. Natl. Acad. Sci. USA 2014, 111, 13217-13222. [CrossRef] [PubMed]

12. Lee, Y.; Dominy, J.E.; Choi, Y.J.; Jurczak, M.J.; Tolliday, N.; Camporez, J.P.; Chim, H.; Lim, J.-H.; Ruan, H.-B.; Yang, X.; et al. Cyclin D1-Cdk4 controls glucose metabolism independently of cell cycle progression. Nat. Cell Biol. 2014, 510, 547-551. [CrossRef] 
13. Steppan, C.M.; Bailey, S.T.; Bhat, S.; Brown, E.J.; Banerjee, R.R.; Wright, C.M.; Patel, H.R.; Ahima, R.S.; Lazar, M.A. The hormone resistin links obesity to diabetes. Nat. Cell Biol. 2001, 409, 307-312. [CrossRef]

14. Vittal, B.; Praveen, G.; Deepak, P. A study of body mass index in healthy individuals and its relationship with Fasting blood sugar. J. Clin. Diagn. Res. 2010, 4, 3421-3424.

15. Friedman, J.M.; Halaas, J.L. Leptin and the regulation of body weight in mammals. Nat. Cell Biol. 1998, 395, 763-770. [CrossRef]

16. Grzemski, A.; Stachowiak, M.; Flisikowski, K.; Mankowska, M.; Krzeminska, P.; Gogulski, M.; Aleksiewicz, R.; Szydlowski, M.; Switonski, M.; Nowacka-Woszuk, J. FTO and IRX3 Genes are Not Promising Markers for Obesity in Labrador Retriever Dogs. Ann. Anim. Sci. 2019, 19, 343-357. [CrossRef]

17. Nishii, N.; Takasu, M.; Soe, O.K.; Maeda, S.; Ohba, Y.; Inoue-Murayama, M.; Kitagawa, H. Cloning, expression and investigation for polymorphisms of canine peroxisome proliferator-activated receptors. Comp. Biochem. Physiol. Part B Biochem. Mol. Biol. 2007, 147, 690-697. [CrossRef]

18. Scherag, A.; Jarick, I.; Grothe, J.; Biebermann, H.; Scherag, S.; Volckmar, A.-L.; Vogel, C.I.G.; Greene, B.; Hebebrand, J.; Hinney, A. Investigation of a Genome Wide Association Signal for Obesity: Synthetic Association and Haplotype Analyses at the Melanocortin 4 Receptor Gene Locus. PLoS ONE 2010, 5, e13967. [CrossRef]

19. Raffan, E.; Dennis, R.J.; O’Donovan, C.J.; Becker, J.M.; Scott, R.A.; Smith, S.P.; Withers, D.J.; Wood, C.J.; Conci, E.; Clements, D.N.; et al. A Deletion in the Canine POMC Gene Is Associated with Weight and Appetite in Obesity-Prone Labrador Retriever Dogs. Cell Metab. 2016, 23, 893-900. [CrossRef]

20. Mankowska, M.; Krzeminska, P.; Graczyk, M.; Switonski, M. Confirmation that a deletion in the POMC gene is associated with body weight of Labrador Retriever dogs. Res. Vet. Sci. 2017, 112, 116-118. [CrossRef]

21. Kao, P.Y.; Leung, K.H.; Chan, L.W.; Yip, S.P.; Yap, M.K. Pathway analysis of complex diseases for GWAS, extending to consider rare variants, multi-omics and interactions. Biochim. Biophys. Acta Gen. Subj. 2017, 1861, 335-353. [CrossRef]

22. Tam, V.; Patel, N.; Turcotte, M.; Bossé, Y.; Paré, G.; Meyre, D. Benefits and limitations of genome-wide association studies. Nat. Rev. Genet. 2019, 20, 467-484. [CrossRef] [PubMed]

23. Dadousis, C.; Pegolo, S.; Rosa, G.J.M.; Bittante, G.; Cecchinato, A. Genome-wide association and pathway-based analysis using latent variables related to milk protein composition and cheesemaking traits in dairy cattle. J. Dairy Sci. 2017, 100, 9085-9102. [CrossRef]

24. Srikanth, K.; Lee, S.-H.; Chung, K.-Y.; Park, J.-E.; Jang, G.-W.; Park, M.-R.; Kim, N.Y.; Kim, T.-H.; Chai, H.-H.; Park, W.C.; et al. A Gene-Set Enrichment and Protein-Protein Interaction Network-Based GWAS with Regulatory SNPs Identifies Candidate Genes and Pathways Associated with Carcass Traits in Hanwoo Cattle. Genes 2020, 11, 316. [CrossRef]

25. Dadousis, C.; Pegolo, S.; Rosa, G.J.M.; Gianola, D.; Bittante, G.; Cecchinato, A. Pathway-based genome-wide association analysis of milk coagulation properties, curd firmness, cheese yield, and curd nutrient recovery in dairy cattle. J. Dairy Sci. 2017, 100, 1223-1231. [CrossRef]

26. Lin, P.-L.; Yu, Y.-W.; Chung, R.-H. Pathway Analysis Incorporating Protein-Protein Interaction Networks Identified Candidate Pathways for the Seven Common Diseases. PLoS ONE 2016, 11, e0162910. [CrossRef]

27. Usui, S.; Yasuda, H.; Koketsu, Y. Characteristics of obese or overweight dogs visiting private Japanese veterinary clinics. Asian Pac. J. Trop. Biomed. 2016, 6, 338-343. [CrossRef]

28. Club, A.K. The Complete Dog Book; Howell Books: New York, NY, USA, 1998.

29. Plassais, J.; Rimbault, M.; Williams, F.J.; Davis, B.W.; Schoenebeck, J.J.; Ostrander, E.A. Analysis of large versus small dogs reveals three genes on the canine $\mathrm{X}$ chromosome associated with body weight, muscling and back fat thickness. PLoS Genet. 2017, 13, e1006661. [CrossRef]

30. Kang, J.M.; Seo, D.; Lee, S.H.; Lee, D.H.; Kim, Y.K.; Choi, B.H.; Lee, S.-H. Genome-wide association study to identify canine hip dysplasia loci in dogs. J. Anim. Sci. Technol. 2020, 62, 306-312. [CrossRef]

31. Pegolo, S.; Mach, N.; Ramayo-Caldas, Y.; Schiavon, S.; Bittante, G.; Cecchinato, A. Integration of GWAS, pathway and network analyses reveals novel mechanistic insights into the synthesis of milk proteins in dairy cows. Sci. Rep. 2018, 8, 1-15. [CrossRef] [PubMed]

32. Lee, E.; Takita, C.; Wright, J.L.; Slifer, S.H.; Martin, E.R.; Urbanic, J.J.; Langefeld, C.D.; Lesser, G.J.; Shaw, E.G.; $\mathrm{Hu}, \mathrm{J} . J$. Genome-wide enriched pathway analysis of acute post-radiotherapy pain in breast cancer patients: A prospective cohort study. Hum. Genom. 2019, 13, 28. [CrossRef] [PubMed] 
33. Lund, E.M.; Armstrong, P.J.; Kirk, C.A.; Klausner, J.S. Prevalence and risk factors for obesity in adult dogs from private US veterinary practices. Int. J. Appl. Res. Vet. Med. 2006, 4, 177-186.

34. Antalis, T.M.; Bugge, T.H.; Wu, Q. Membrane-Anchored Serine Proteases in Health and Disease. Prog. Mol. Biol. Transl. Sci. 2011, 99, 1-50. [CrossRef]

35. Hachem, J.-P.; Houben, E.; Crumrine, D.; Man, M.-Q.; Schurer, N.Y.; Roelandt, T.; Choi, E.H.; Uchida, Y.; Brown, B.E.; Feingold, K.R.; et al. Serine Protease Signaling of Epidermal Permeability Barrier Homeostasis. J. Investig. Dermatol. 2006, 126, 2074-2086. [CrossRef]

36. Böttcher-Friebertshäuser, E. Membrane-Anchored Serine Proteases: Host Cell Factors in Proteolytic Activation of Viral Glycoproteins. In Activation of Viruses by Host Proteases; Springer: Cham, Switzerland, 2018; pp. 153-203.

37. Marks, D.F. Homeostatic theory of obesity. Health Psychol. Open 2015, 2, 1-30. [CrossRef]

38. Smith, A.K.; Fang, H.; Whistler, T.; Unger, E.R.; Rajeevan, M.S. Convergent Genomic Studies Identify Association of GRIK2 and NPAS2 with Chronic Fatigue Syndrome. Neuropsychobiology 2011, 64, 183-194. [CrossRef]

39. Delgado, T.C. Glutamate and GABA in Appetite Regulation. Front. Endocrinol. 2013, 4, 103. [CrossRef] [PubMed]

40. Zhou, X.; Wang, W.; Zhang, S.; Wang, X.; Tang, Z.; Gu, J.; Li, J.; Huang, J. CACNA1B (Cav2. 2) overexpression and its association with clinicopathologic characteristics and unfavourable prognosis in non-small cell lung cancer. Dis. Markers 2017, 2017, 6136401. [CrossRef]

41. Wang, C.-Y.; Lai, M.-D.; Phan, N.N.; Sun, Z.; Lin, Y.-C. Meta-Analysis of Public Microarray Datasets Reveals Voltage-Gated Calcium Gene Signatures in Clinical Cancer Patients. PLoS ONE 2015, 10, e0125766. [CrossRef]

42. Zhai, M.; Yang, D.; Yi, W.; Sun, W. Involvement of calcium channels in the regulation of adipogenesis. Adipocyte 2020, 9, 132-141. [CrossRef]

43. Tao, Y.-X. The Melanocortin-4 Receptor: Physiology, Pharmacology, and Pathophysiology. Endocr. Rev. 2010, 31, 506-543. [CrossRef]

44. Ross, S.E.; Hemati, N.; Longo, K.A.; Bennett, C.N.; Lucas, P.C.; Erickson, R.L.; MacDougald, O.A. Inhibition of Adipogenesis by Wnt Signaling. Science 2000, 289, 950-953. [CrossRef]

45. Fuster, J.J.; Zuriaga, M.A.; Ngo, D.T.-M.; Farb, M.G.; Aprahamian, T.R.; Yamaguchi, T.P.; Gokce, N.; Walsh, K. Noncanonical Wnt Signaling Promotes Obesity-Induced Adipose Tissue Inflammation and Metabolic Dysfunction Independent of Adipose Tissue Expansion. Diabetes 2015, 64, 1235-1248. [CrossRef]

46. Chen, N.; Wang, J. Wnt/ß-Catenin Signaling and Obesity. Front. Physiol. 2018, 9, 792. [CrossRef]

47. Grant, S.F.A.; Thorleifsson, G.; Reynisdottir, I.; Benediktsson, R.; Manolescu, A.; Sainz, J.; Helgason, A.; Stefansson, H.; Emilsson, V.; Helgadottir, A.; et al. Variant of transcription factor 7-like 2 (TCF7L2) gene confers risk of type 2 diabetes. Nat. Genet. 2006, 38, 320-323. [CrossRef] [PubMed]

48. Dissanayake, W.C.; Sorrenson, B.; Shepherd, P.R. The role of adherens junction proteins in the regulation of insulin secretion. Biosci. Rep. 2018, 38, 38. [CrossRef]

49. Shi, C.; Zhang, M.; Tong, M.; Yang, L.; Pang, L.; Chen, L.; Xu, G.; Chi, X.; Hong, Q.; Ni, Y.; et al. miR-148a is Associated with Obesity and Modulates Adipocyte Differentiation of Mesenchymal Stem Cells through Wnt Signaling. Sci. Rep. 2015, 5, srep09930. [CrossRef] [PubMed]

50. Stone, T.W.; McPherson, M.; Darlington, L.G. Obesity and Cancer: Existing and New Hypotheses for a Causal Connection. EBioMedicine 2018, 30, 14-28. [CrossRef]

51. Lim, H.-Y.; Im, K.-S.; Kim, N.-H.; Kim, H.-W.; Shin, J.-I.; Yhee, J.-Y.; Sur, J.-H. Effects of obesity and obesity-related molecules on canine mammary gland tumours. Vet. Pathol. 2015, 52, 1045-1051. [CrossRef]

52. Thun, G.A.; Imboden, M.; Berger, W.; Rochat, T.; Probst-Hensch, N.M. The Association of a Variant in the Cell Cycle Control Gene CCND1 and Obesity on the Development of Asthma in the Swiss SAPALDIA Study. J. Asthma 2013, 50, 147-154. [CrossRef] [PubMed]

53. Ding, L.; Zhang, L.; Biswas, S.; Schugar, R.C.; Brown, J.M.; Byzova, T.V.; Podrez, E.A. Akt3 inhibits adipogenesis and protects from diet-induced obesity via WNK1/SGK1 signalling. JCI Insight 2017, 2, e95687. [CrossRef]

54. Yang, X.; Zhu, H.; Qin, Q.; Yang, Y.; Yang, Y.; Cheng, H.; Sun, X. Genetic variants and risk of esophageal squamous cell carcinoma: A GWAS-based pathway analysis. Gene 2015, 556, 149-152. [CrossRef] [PubMed] 
55. Zhang, J.-W.; Klemm, D.J.; Vinson, C.; Lane, M.D. Role of CREB in Transcriptional Regulation of CCAAT/Enhancer-binding Protein $\beta$ Gene during Adipogenesis. J. Biol. Chem. 2004, 279, 4471-4478. [CrossRef]

56. Ye, J. Mechanisms of insulin resistance in obesity. Front. Med. 2013, 7, 14-24. [CrossRef]

57. Song, Z.; Wang, Y.; Zhang, F.; Yao, F.; Sun, C. Calcium Signaling Pathways: Key Pathways in the Regulation of Obesity. Int. J. Mol. Sci. 2019, 20, 2768. [CrossRef]

58. Kaser, S.; Sandhofer, A.; Föger, B.; Ebenbichler, C.F.; Igelseder, B.; Malaimare, L.; Paulweber, B.; Patsch, J.R. Influence of obesity and insulin sensitivity on phospholipid transfer protein activity. Diabetologia 2001, 44, 1111-1117. [CrossRef]

Publisher's Note: MDPI stays neutral with regard to jurisdictional claims in published maps and institutional affiliations.

(C) 2020 by the authors. Licensee MDPI, Basel, Switzerland. This article is an open access article distributed under the terms and conditions of the Creative Commons Attribution (CC BY) license (http://creativecommons.org/licenses/by/4.0/). 